\title{
Practical Considerations in Adopting New Technology for Facial Cosmetic Procedures
}

\author{
Joseph J. Rousso, $\mathrm{MD}^{1,2}$ \\ ${ }^{1}$ Manhatten Facial Plastic Surgery and ENT, New York, New York \\ 2 Department of Otolaryngology - Head \& Neck Surgery, Icahn School \\ of Medicine at Mount Sinai, New York, New York
}

Address for correspondence Joseph J. Rousso, MD, Manhatten Facial Plastic Surgery and ENT, 247 Third Avenue, Suite 404, New York, NY 10010 (e-mail: manhattanfacialplasticdoc@gmail.com).

Facial Plast Surg 2020;36:684-687.

\author{
Abstract \\ Keywords \\ - facial plastic surgery \\ - emerging \\ technologies \\ - minimally invasive \\ - facelift \\ - face tightening
}

The field of facial plastic and reconstructive surgery encompasses both surgical and nonsurgical facets, creating a supplemental level of care and an additional layer of complexity. Determining the "best course of care" can be very difficult in experienced situations, but even more so when considering adopting an emerging technology. A basic and practical method of analyzing a new technology requires investigating the risk-tobenefit assessment, the utility and clinical outcomes compared with other treatment options, and an introspective ethical appraisal of whether the technology is foremost for patient care purposes. Even after employing a new technology, constant monitoring and reevaluation of the results is necessary to determine if it should be continued or altered.
The practice of facial plastic surgery lends itself more completely to a scientific art form than, arguably, any other field of medicine. Several factors come into play when deciding the most optimal course of action in any respective clinical situation. And, like the most complicated and integral fields, each variable can have its own set of consequences.

Facial plastic and reconstructive surgery (FPRS) includes both surgical and nonsurgical aspects, adding both a secondary arsenal of care and an additional layer of complexity in decision-making for the best course of action. A patient who presents with an aesthetic nasal complaint can be presented with a variety of options ranging from less invasive filler sculpting to various types of rhinoplasty approaches; oftentimes, patients bring specific requests such as a particular type of filler for a "liquid rhinoplasty" or an off-label use of a new laser device. These sorts of situations and several others require sober-minded approaches to determine the best and safest way to approach and satisfy patient goals and wishes. This is particularly true when incorporating a new tool into the care plan.

In our field, a series of options can exist for patient complaints or desire. The decision process in determining the "best course" of action is a complex one and may seem mired by situational settings. A discussion of factors that come into play when assessing and determining the best options, in the evaluation of emerging technologies and treatments, is the objective of this article. A basic and practical method of analyzing a new technology would include identifying and investigating questions that relate to the risk/benefit assessment, utility, and outcomes compared with others. In addition, an exclusive set of introspective questions should be asked in regard to the ethical dilemmas that may arise from incorporating a new technology into your practice. Finally, the informed consent is the most crucial part of the determination of whether or not to employ an emerging technology in each specific case.

\section{Risk-Benefit Assessment}

\section{Risk Analysis}

In practical terms, the author begins with assessing the risks and possible untoward consequences. This is the most consistent method following the overarching physician tenet, "first do no harm." Familiarize yourself with all of the possible complications and negative aspects. Be prepared for situations where your consideration does not go beyond this stage, and
Issue Theme Technologic Advancements in Head and Neck Reconstruction; Guest Editors: Sameep P. Kadakia, MD, and Sunishka M. Wimalawansa, MD, MBA
Copyright $\odot 2020$ by Thieme Medical Publishers, Inc., 333 Seventh Avenue, New York, NY 10001, USA. Tel: +1(212) 760-0888. 
certain risks should be considered beyond the comfort zone of the surgeon to consider proceeding.

An up-to-date empirical analysis of data that would allow the surgeon to determine and assess the risk-to-benefit profile should be developed. For medical devices, use the Food \& Drug Administration's (FDA) up-to-date medical device safety Web site https://www.fda.gov/medical-devices/medical-device-safety. This can help to determine if there are any glaring risks or recent warnings about the device in question. A search for the name of the device or technology on the FDA Web site may help gather more information. Any liabilities that are listed should be further investigated and considered a very serious risk. Similarly, for medications or biologic products, the FDA Web site should be visited to determine if there is any additional information such as medication guides.

Next in the thought process is a focused literature search for knowledge of any and every possible adverse consequence that could occur. These focused searches are not as straightforward as one would imagine. PubMed searches are an excellent way to begin; use of key terms such as "adverse effects," "risks," or "complications" in association with the type of technology is often more productive than searching a specific brand or product name. For example, prior to incorporating resurfacing lasers into one's practice, a PubMed search of "complications of resurfacing lasers" gives a variety of information that could be thoroughly reviewed. These articles include literature reviews of complications, ${ }^{1}$ comparisons of the downtime and effectiveness of different types of resurfacing and fractional lasers, ${ }^{2}$ and assessment articles of the current state of resurfacing. ${ }^{3}$ This is a very time-consuming and difficult exercise but one that is a core necessity to gain insight. Additionally, this can be a very biased exercise if one chooses not to go through all of the available information and assess it with appropriate credence insofar as its level of evidence is concerned. It should be noted that these focused literature searches may also provide further information such as utility and efficacy.

Next, trusted colleagues and mentors, who do not have conflicts of interest with your practice, are a great source of reliable information. These colleagues would ideally be ones who have direct experience with the same or similar technology. What they can potentially offer is an honest opinion about any complications that they did not consider when they purchased or began utilizing the new technology.

Finally, local and regional specialty society meetings are a great place to have an open conversation about risks associated with new technologies. However, close attention should be focused on the sources of information that you use and trust. In particular, sponsorships of meetings by companies or colleagues who may have a vested interest should be ascertained by noting financial disclosures.

At this point, you should have established a safety profile based on the extensive risk analysis that you conducted.

\section{Benefit Evaluation}

This is generally the easiest part of your research. In particular, manufacturers of the product or technology will emphasize what the benefits are and routinely provide positive studies or data accordingly. Although this may be helpful, it should be assessed with a careful level of skepticism on account of the one-sidedness that may be provided.

Although patients tend to ask for particular products, the benefits that they are often presented may often seem overly ambitious.

One objective method of benefit determination, in our field, is photo analysis. You may be presented with before and after photos of a minimally invasive radiofrequency device that promotes skin tightening/facial rejuvenation. Alternatively, you may be presented with literature on a new injectable filler whose viscosity is claimed to be ideal for lip augmentation and given molecular composition data to support this. Either of these situations requires you to be a clinical investigator and determine whether or not this makes sense. Photos can be deceiving if they do not follow standardized methods, and data on the properties of an injectable require attaining a certain knowledge base prior to making an informed decision (i.e., an understanding of the rheology involved in different fillers).

An additional source of excellent information comes from the American Academy of Facial Plastic and Reconstructive Surgery's (AAFPRS) Emerging Trends \& Technology Committee. Their stated goal is to "review and disseminate information on innovations in treatment, surgical procedures, implants and other devices; recommend policies relating to surgical techniques and devices in the interest of providing the best possible care for patients" 4

At this point, you can formulate a risk-benefit assessment. This can be quite difficult because individual clinicians have unique perspectives. It must be emphasized that the role of determining the risk-to-benefit profile is on the physician and should be explained to the patient with full disclosure in understandable terminology for informed consent (discussed further in the Ethical Considerations section). FPRS practitioners are accustomed to situations where patients have an excellent knowledge of what they want; however, it should be understood that a lot of the information that a patient may have on new technologies/products is often based on direct to consumer advertising. In a 2018 study that sought to evaluate the risk versus benefit information provided in television ads for prescription drugs, Fahim et al determined that a lack of consistency exists and even found that a large number of those with black box warnings did not communicate these in advertising. ${ }^{5}$ This highlights the point that sufficient risk-tobenefit information is not always available to our patients without direct physician input. Because there is no universally accepted exact formula for the risk-to-benefit ratio that is acceptable for adopting a new technology, a detailed explanation to the patient can help them determine their wishes and assess what is acceptable to them.

\section{Utility and Outcomes}

Several factors are involved in determining whether or not there is utility in employing a new technology. First, the practitioner has to determine whether it will add a useful instrument to their arsenal of care and/or whether it can hinder their other tools. As surgeons, we often consider 
surgical intervention as the highest yield method for achieving maximal results, albeit understanding that this is the more invasive approach. Cosmetic surgeries such as rhinoplasty, facelift, necklift, and blepharoplasty are generally considered the gold standards for facial enhancement. ${ }^{6}$ Accordingly, we may tend to consider effective newer technologies as "temporizing measures" to achieve some positive results with less risk than surgery until such time that the patient would prefer to pursue surgery.

Certainly, a very useful new technology is one that would offer improvements without hindering any future surgical care. As an example, I have offered patients microfocused ultrasound with visualization (MFU-V) tightening device procedures as a nonsurgical option to face and neck rejuvenation over the past 8 years. I found that many patients came requesting this procedure or had this done elsewhere and wanted to consider additional treatments. Though generally positive (particularly in those with lower body mass index), the improvement in skin laxity varied at all ages, as consistent

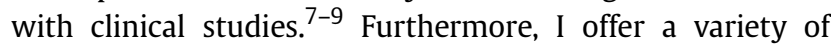
surgical rhytidectomy procedures tailored to individual patients. These face and neck lifts span the gamut from plication with local anesthesia to comprehensive deep plane rhytidectomy under general anesthesia. I found that all of these surgical options were somewhat altered in patients who had thermal tightening procedures. Specifically, the dissection and elevation of tissue planes were more akin to those of revision rhytidectomy procedures, secondary to the thick scar tissue formed. This led to longer surgical time and more difficult rhytidectomies than what would be expected in a typical primary aging face patient. This was a finding that was anecdotally confirmed by several colleagues who have performed similar surgeries. I came to understand that although there are benefit and utility to the emerging technology of heat-based skin tightening devices, there is an element of deterrence to the tool that I consider the gold standard: rhytidectomy. Nonetheless, the technologically advanced minimally invasive devices do play a significant role in several situations both as a temporizing measure in certain cases and as an instrument of comprehensive care in patients who do not plan on undergoing surgery. They add utility in a variety of settings and certainly add to the armamentarium of the facial plastic surgeon.

Second, in determining utility is a physician examination of the cost-effectiveness of employing an emerging technology. The answer to this is very intertwined in the likely settings and patient cohorts that would optimally benefit from this procedure. In keeping with the prior example, a determination of how many total patients in your practice would benefit from a new thermal tightening procedure at a particular price point as compared with the associated costs. To consider this fully, one must start by examining the capital costs, time/opportunity costs to the physician and their employees, maintenance and disposable costs, and, of course, the total cost to the patient. The capital investment involved in purchasing a new thermal skin tightening device can be upwards of US $\$ 100,000$. A minimum of 1 hour of time is required for an effective procedure, and additional time for numbing, patient experience, and comfort factors. Depending on the setting of your practice, this may be the time that is largely occupied by the physician themselves and/or an additional essential employee. Disposable costs can range from hundreds to thousands of dollars per procedure. Patient cost per treatment can vary in range but would need to be priced in the thousands of dollars per treatment range just to cover overhead. Offering these sorts of treatments on occasion is not necessarily cost-effective to a facial plastic surgery practice, whereas more routine use in the correct patient population would make the most sense. Although there are options to try to minimize capital costs such as leasing devices, there should also be consideration of having a reliable subspecialist whom you can trust to refer these procedures to. Over the years, I have personally evolved from trying to offer my patients every possible service to appreciating that there is significant merit and cost-effectiveness in focusing largely on the surgical interventions that I consider the gold standards, and relying on expert, trusted nonsurgical colleagues to offer my patients advanced minimally invasive procedures.

Third, even after deciding to employ the new technology, there should be a constant and up-to-date analysis of outcomes performed by the physician. As physicians, we strive to be endless learners, and there is no better way to achieve this than to study and question our own outcomes. Analyzing and reanalyzing long-term results, standardized before and after photos, and patient experience surveys help achieve the best possible outcomes. These are methods of gathering data that may help improve and, in some instances, may lead to changing or abandoning a technology. Further analysis in comparison with the rest of our surgical arsenal can help us give our patients the most accurate presentation of options. For example, after analyzing results of thermal tightening devices used over several years in comparison to my deep plane rhytidectomy results over those same years, I came to realize that in the very best-case scenarios the results were approximately $15 \%$ as effective. This was approximately 10 to $20 \%$ less effective than I had previously realized, and I would not have been able to learn this without constant comparative analysis. In fact, one of the most common questions that I receive from patients is one of comparing the effectiveness of thermal tightening devices to facelift surgery. I make it very clear that there is no exact science to determining this, but my estimations are based on self-analyzing prior results and that in my hands, in the best-case scenarios, they are approximately $15 \%$ as effective as a surgical rhytidectomy.

At this point, you have determined that the risk-to-benefit profile is favorable, there is utility in employing this emerging technology, and you anticipate advantageous outcomes. An ethical analysis of whether adopting the technology in question will be foremost for the benefit of the patient is the next step.

\section{Ethical Considerations}

This may be the most difficult of considerations and requires a significant amount of clinician introspection to perform effectively. Three specific ethical situational considerations 
should be examined: business model, unrecognized health risks, and informed consent.

\section{Cosmetic Business Models}

Cosmetic practitioners earn their income and livelihood from performing elective consultations and procedures for direct payment. There is no third-party involvement that approves or denies a procedure based on medical necessity, as is the case with common functional procedures. This can potentially, even subconsciously, create an inherent bias in the treatment options our patients are offered. Atiyeh et al describe that "aesthetic surgery that works only according to market categories runs the risk of losing the view of the real need of patients ... aims to sell something, not to help people." ${ }^{10}$ By their very nature, inherent economic biases can make the clinician feel that they are promoting the best option by offering a recently acquired emerging technology to a patient rather than a less expensive and less glamorous option. This is particularly true if the cost-effectiveness of incorporating a new technology into your practice requires a large volume of use. A constant self-reflection that involves asking yourself as a scientist and clinician to eliminate the business aspects involved and determine which option is the unique patient in front of you most likely to benefit the most from.

\section{Unrecognized Health Risks}

Emerging technologies are so named because they are by definition "becoming apparent." Though there is a positive and advantageous connotation associated with emerging technologies, there are also potential emerging risks that may not become apparent until they have had sufficient time to declare themselves. The recent history of aesthetic medicine has proven time and time again that some treatments hailed as advanced beautification techniques turned out to have terrible consequences. One example is that of facial injectables for volume restoration. Paraffin injections were utilized in different parts of the world for decades in attempts to restore facial youthfulness and caused delayed, severe, and debilitating complications. Public anger to these complications was so great that historians believed this to have slowed the advancement of cosmetic surgery. ${ }^{11}$ Even modern-day hyaluronic acid fillers, whose safety profiles we often tout as excellent, have the potentially life-altering risks of vascular compromise, skin necrosis, and blindness. Though there is no way to predict the future and determine what will and what will not cause previously unrecognized consequences, watchful waiting for a short period of time can be paramount to avoiding serious risk. I often counsel patients and trainees that being one of the first to undergo or use an emerging technology may be academically exciting but practically problematic.

\section{Informed Consent}

The most important ethical aspect of performing an elective procedure is patient consent. There are subtle discrepancies between the definition of "consent" and that of "informed consent," but these play a particularly important role in plastic surgery. ${ }^{10,12}$ For a patient to make an "informed" decision and consent, they often rely on the physician explanation. However, when a new technology is involved, there is simply limited information. Practical and important discussion points include an honest assessment of your research of and overall experience, no matter how limited, with this new technology. Certainly, a clear explanation risk-to-benefit analysis, attempted goals of treatment, and alternatives of care are essential components.

\section{Conclusion}

Employing emerging technologies into your practice can be very helpful to your patients and professionally rewarding. These can open up avenues of growth in your practice and can allow you to offer more alternatives to patient care. However, prior to adopting any new technology, a comprehensive and focused assessment needs to be performed. Riskto-benefit analysis, utility, clinical outcomes, and ethical considerations should be the cornerstones of your focused assessment. If and when the decision to employ an emerging technology into your practice is made, constant monitoring and reevaluations over time should be performed to assure that it is foremost achieving the goal of benefiting patients.

Conflict of Interest

None declared.

\section{References}

1 Li D, Lin SB, Cheng B. Complications and posttreatment care following invasive laser skin resurfacing: a review. J Cosmet Laser Ther 2018;20(03):168-178

2 You HJ, Kim DW, Yoon ES, Park SH. Comparison of four different lasers for acne scars: Resurfacing and fractional lasers. J Plast Reconstr Aesthet Surg 2016;69(04):e87-e95

3 Carniol PJ, Hamilton MM, Carniol ET. Current Status of Fractional Laser Resurfacing. JAMA Facial Plast Surg 2015;17(05):360-366

4 AAFPRS. Academy Committees. Available at: https://www.aafprs. org/Professionals/Membership/Academy_Committees.aspx. Accessed on September 15, 2020

5 Fahim G, Toscani M, Barone JA, Wang C, Gandhi S. Evaluation of risk versus benefit information in direct-to-consumer (DTC) prescription drug television advertisements. Ther Innov Regul Sci 2018;52(01):114-117

6 Devgan L, Singh P, Durairaj K. Surgical cosmetic procedures of the face. Otolaryngol Clin North Am 2019;52(03):425-441

7 Alhaddad M, Wu DC, Bolton J, et al. A randomized split face, evaluator blind clinical trial comparing monopolar radiofrequency versus microfocused ultrasound with visualization for lifting and tightening of the face and upper neck. Dermatol Surg 2019;45(01):131-139

8 Oni G, Hoxworth R, Teotia S, Brown S, Kenkel JM. Evaluation of a microfocused ultrasound system for improving skin laxity and tightening in the lower face. Aesthet Surg J 2014;34(07):1099-1110

9 Fabi SG, Goldman MP. Retrospective evaluation of micro-focused ultrasound for lifting and tightening the face and neck. Dermatol Surg 2014;40(05):569-575

10 Atiyeh BS, Rubeiz MT, Hayek SN. Aesthetic/cosmetic surgery and ethical challenges. Aesthetic Plast Surg 2008;32(06):829-839, discussion 840-841

11 Kim JE, Sykes JM. Hyaluronic acid fillers: history and overview. Facial Plast Surg 2011;27(06):523-528

12 O'Brien CM, Thorburn TG, Sibbel-Linz A, McGregor AD. Consent for plastic surgical procedures. J Plast Reconstr Aesthet Surg 2006; 59(09):983-989 